\title{
TRABAJO, GÉNERO Y NUEVAS TECNOLOGÍAS: ALGUNAS CONSIDERACIONES
}

\author{
Ana María Romero Burillo \\ Profesora Titular de Universidad de Derecho del Trabajo y de la Seguridad Social. \\ Universitat de Lleida
}

\begin{abstract}
Este trabajo analizará desde una perspectiva de género el impacto de las NTICs en aspectos vinculados a las tres fases principales del desarrollo de la relación laboral: en primer lugar, en la fase del acceso al mercado de trabajo, realizando un análisis de las incidencia que tienen las NITCs en la intermediación privada y, concretamente, el uso que se realiza de las plataformas digitales desde una perspectiva de género; en segundo lugar, en la fase de permanencia de la relación de trabajo, con el análisis de las ventajas e inconvenientes que puede ofrecer el teletrabajo a la hora de conciliar la vida personal, familiar y laboral; y, en tercer lugar, en la fase de extinción del contrato de trabajo, con el estudio del papel que pueden jugar los nuevos sistemas de comunicación en supuestos de acoso sexual y por razón de sexo en el entorno laboral.
\end{abstract}

This paper will analyze from a gender perspective the impact of NICTs in aspects related to the three main phases of the development of the employment relationship: first, in the phase of access to the labor market, performing an analysis of the impact that the NITCs have on the intermediation private sector and, specifically, the use that is made of digital platforms from a gender perspective; secondly, in the phase of permanence of the employment relationship, with the analysis of the advantages and disadvantages that teleworking can offer when it comes to reconciling personal, family and work life; and, thirdly, in the phase of termination of the employment contract, with the study of the role that new communication systems can play in cases of sexual harassment and because of sex in the workplace.

IUSLabor 1/2019, ISSN 1699-2938, p. 210-232

DOI. 10.31009/IUSLabor.2019.i01.04

Title: Work, gender and new technologies: some considerations

Palabras clave: nuevas tecnologías, relación de trabajo, intermediación, teletrabajo, conciliación personal, familiar y laboral y ciberacoso sexual.

Key words: new technologies, work relation, intermediation, telework, reconciliation personal, family and work life and sexual cyberbullying. 


\section{Sumario}

1. Presentación del tema

2. La incidencia de las NTICs en la incorporación de la mujer en el mercado de trabajo

3. El teletrabajo y la conciliación de la vida personal, familiar y profesional

4. El papel de las NTICs en los supuestos de acoso sexual y por razón de sexo: el ciberacoso

5. Bibliografía 


\section{Presentación del tema}

El estudio del impacto que la innovación tecnológica tiene en el ámbito de las relaciones de trabajo es algo consustancial a la disciplina del Derecho del Trabajo. No en vano uno de los factores que intervienen en la transformación de las relaciones de trabajo y en la consiguiente aparición de las primeras normas laborales es justamente el fulgurante desarrollo técnico que se produce a partir del último tercio del siglo XVIII y que conocemos como Revolución Industrial. A este primer gran avance técnico le han seguido otros muchos que, progresivamente, han llevado a la transformación de la configuración inicial de las figuras de trabajador/trabajadora y empresario/empresaria, así como del modo de trabajar y de entender las relaciones laborales, requiriendo para ello de la continua adaptación de nuestro ordenamiento jurídico-laboral.

Ahora bien, convenimos con la doctrina iuslaboralista ${ }^{1}$ en que los cambios tecnológicos que se están produciendo en la actualidad, aunque tienen puntos en común con anteriores "revoluciones tecnológicas" acaecidas en los dos últimos siglos, cuentan a su vez con características propias que hacen que tales cambios desarrollen unos efectos sobre la organización del trabajo y los recursos humanos sin precedentes.

Este desarrollo tecnológico al que nos referimos y que de forma tan trascendente está influyendo en la normativa laboral, no sólo abarca al conjunto de innovaciones técnicas que son ideadas para su introducción en las empresas con la finalidad de potenciar su productividad, sino que desde hace ya algunos años, a todas estas innovaciones se han sumado otro tipo de novedades tecnológicas no pensadas específicamente para su uso en las empresas pero que por sus implicaciones sociales inevitablemente han ido progresivamente incrementando su influencia en las relaciones de trabajo, dando lugar a lo que se califica como el "carácter dual" de las nuevas tecnologías ${ }^{2}$.

En este sentido el desarrollo que en la última década se ha producido en el ámbito de las tecnologías de la información y la comunicación (en adelante TICs) ha desembocado en la configuración de un nuevo modelo social de las comunicaciones personales, en donde ya no sólo el correo electrónico ha sustituido prácticamente a cualquier forma de

\footnotetext{
${ }^{1}$ Vid. en este sentido, entre otros CRUZ VILLALÓN, J., "Las transformaciones de las relaciones laborales ante la digitalización de la economía", Temas Laborales, n 118, 2016, p. 13 y ss.; DEL REY GUANTER, S., "Sobre el futuro del trabajo: modalidades de prestaciones de servicios y cambios tecnológicos", IUSLabor, $\mathrm{n}^{\circ}$ 2, 2017, p. 1 a 3. y MERCADER UGUINA, J. R., "La robotización y el futuro del trabajo", Trabajo y Derecho, no 27, 2017, p. 14 y 15.

2 Vid. Falguera BARÓ, M., "Nuevas tecnologías y trabajo (I): perspectiva contractual", Trabajo y Derecho, no 19-20, 2017, p. 33.
} 
comunicación epistolar ${ }^{3}$, sino que también y sobre todo el teléfono móvil, a través de aplicaciones como el "WhatsApp", ha desbancado a los servicios de mensajes cortos (SMS) que hace relativamente poco tiempo eran un medio revolucionario y que ahora, sin embargo, algunos no dudarían en calificarlo de instrumento desfasado de comunicación, sin olvidar, por supuesto, la vertiginosa irrupción de una serie de plataformas y aplicaciones informáticas muy variadas, las llamadas "redes sociales", entre las que se encuentran algunas tan conocidas como Facebook, Twitter, Instagram, LinkedIn, twenti, myspace, google+, etcétera, las cuales actualmente sirven de cauce de comunicación entre un gran número de personas, lo que permite afirmar que estos instrumentos se han incorporado a la vida cotidiana de la población mundial.

A este respecto, a título de ejemplo, según un informe publicado sobre el uso a nivel mundial de las nuevas tecnologías de la información y comunicación (en adelante NTICs), en el año $2017^{4}$, de los 7,5 mil millones de personas que conformaban la población mundial, 3,5 mil millones eran usuarias de internet, 2,7 mil millones eran usuarias de redes sociales y en el último año el número de usuarios de internet se incrementó en un 10\%, lo cual supone la existencia de 354 millones más de usuarios. En el marco de estos datos generales, cabe indicar que casi un cuarto de la población mundial $(22,9 \%)$ utilizaba Facebook, Twiter contaba con 328 millones de usuarios activos al mes a nivel mundial, LinkedIn tenía registrados 467 millones de perfiles y, 1,3 mil millones de personas utilizaban YouTube.

En España 22,4 millones de personas eran usuarias de internet, de las cuales 11,2 millones usaban las redes sociales. Facebook era la red social por excelencia (91\%), seguido de "WhatsApp" (89\%), YouTube (71\%) y Twiter (50\%). En cuanto a los dispositivos más utilizados para la conexión a las redes sociales, el móvil lideraba el ranking (94\%), seguido del ordenador (93\%) y de la Tablet (55\%), si bien este último dispositivo tuvo un auge creciente respecto al año 2016, donde el porcentaje de utilización era del $28 \%$.

El uso masivo y cotidiano de todos estos medios e instrumentos de comunicación ha hecho inevitable que progresivamente se haya introducido dicho uso en las empresas, lo cual ha incidido directamente en el modelo de organización y gestión, con indudables ventajas a estos efectos a nivel estratégico, de gestión del conocimiento, de innovación

\footnotetext{
${ }^{3}$ Selma Penalva, A., "La información reflejada en las redes sociales y su valor como prueba en el proceso laboral. Análisis de los últimos criterios jurisprudenciales", Revista General del Derecho del Trabajo y de la Seguridad Social, no 39, 2014, p. 356.

${ }^{4} \mathrm{Vid}$. https://www.brandwatch.com/es/2017/09/98-estadisticas-de-las-redes-sociales-para-2017/ [Fechas de consulta: 4/06/2018].

${ }^{5}$ Vid. https://iabspain.es/wp-content/uploads/iab_estudioredessociales_2017_vreducida.pdf [Fechas de consulta: 4/06/2018].
} 
y desarrollo, de colaboración, de formación, de captación de talento y sistema de reclutamiento de personal, de comunicación interna y marketing, etcétera. De igual forma, los cambios tecnológicos actuales pueden ofrecer ciertas ventajas a las personas trabajadoras, muchas de ellas coincidentes con las ofrecidas a las empresas, entre las que se pueden mencionar la ampliación y mejora de las ofertas de trabajo, la flexibilidad horaria, la mejor compatibilización de vida personal y profesional, etcétera ${ }^{6}$.

Ahora bien, junto al conjunto de ventajas que acompañan al uso de estas NTICs, de igual forma también se plantean riesgos tanto para la empresa como para las propias personas trabajadoras que derivan directamente de su utilización, algunos de los cuales surgen como consecuencia de la tendencia que existe en estos casos a diluirse la frontera entre lo privado y lo público, entre la vida personal y laboral ${ }^{7}$. Efecto de esta confusión surgen, entre otras cuestiones, la prohibición o la restricción de la utilización de dichos instrumentos durante el tiempo y el lugar de trabajo a la persona trabajadora, la legitimidad de la empresa a acceder a dichos medios de comunicación y a utilizar información a la que puede tener acceso gracias a las TICs, así como el valor que debe darse a la misma a efectos disciplinarios ${ }^{8}$.

Como puede observarse, la problemática que se plantea no es en absoluto nueva, al fin y al cabo, se trata de estudiar una vez más cuestiones vinculadas al acceso de las personas trabajadoras al mercado de trabajo y su permanencia en el mismo. Por tanto, se trata de abordar el estudio de un tema clásico, si bien en el marco de una nueva y constantemente cambiante realidad. En este caso, además introducimos en el estudio un elemento que no siempre se tiene en cuenta y que, sin embargo, tiene una gran trascendencia como es la perspectiva de género. Es decir, además de conocer el impacto que las NTICs tienen en las relaciones de trabajo, nos proponemos analizar si ese impacto es igual con independencia del género de la persona trabajadora, o si, por el contrario, los avances tecnológicos que se incorporan en las empresas inciden de forma diferente en función del género.

El estudio que se plantea es ciertamente ambicioso y su desarrollo completo desborda los límites de extensión de un artículo doctrinal, por ello, en este trabajo analizaremos,

\footnotetext{
${ }^{6}$ Vid. en este sentido, CARDONA RUBERT, M., "La utilización de las redes sociales en el ámbito de la empresa", Revista de Derecho Social, nº 52, 2010, p. 68; LLORENS ESPADA, J., "El uso de facebook en los procesos de selección de personal y la protección de los derechos de los candidatos", Revista de Derecho Social, $\mathrm{n}^{\circ}$ 68, 2014; NORES TORRES, L.E., "Algunos puntos críticos sobre la repercusión de las redes sociales en el ámbito de las relaciones laborales: aspectos individuales, colectivos y procesales", Revista de Información Laboral, nº 7, 2016, p. 4 a 8., y TALENS VisCONTI, E. E., "La libertad de expresión de los sindicatos en las redes sociales", Aranzadi Social, versión electrónica, BIB 2015 2271, p. 1.

${ }^{7}$ Vid. CARDONA RuBERT, M. B., "La utilización de las redes sociales...", cit. p. 69 y 70.

${ }^{8}$ Vid. por todos TALENS VisCONTI, E. E., "La libertad de expresión...”, cit. p. 1 y 2.
} 
sin ánimo de exhaustividad, algunos ámbitos que por el alcance e importancia que tienen en el marco de las relaciones de trabajo, resulta especialmente interesante estudiar, para lo cual, además de la normativa laboral reguladora, tomaremos como referencia principal la reciente doctrina que nos ofrecen los órganos judiciales en esta materia.

Concretamente, el estudio analizará el impacto de las NTICs en aspectos vinculados a las tres fases principales del desarrollo de la relación laboral: en primer lugar, en la fase del acceso al mercado de trabajo, realizando un análisis de las incidencia que tienen las NITCs en la intermediación privada y, concretamente, el uso que se realiza de las plataformas digitales desde una perspectiva de género; en segundo lugar, en la fase de permanencia de la relación de trabajo, con el análisis de las ventajas e inconvenientes que puede ofrecer el teletrabajo a la hora de conciliar la vida personal, familiar y laboral; y, en tercer lugar, en la fase de extinción del contrato de trabajo, con el estudio del papel que pueden jugar los nuevos sistemas de comunicación en supuestos de acoso sexual y por razón de sexo en el entorno laboral.

\section{La incidencia de las NTICs en la incorporación de la mujer en el mercado de trabajo}

Las políticas dirigidas a la consecución del pleno empleo es una de las cuestiones que de forma ineludible y permanentemente figuran en las agendas gubernamentales ${ }^{9}$, siendo especialmente importantes en momentos de crisis económica, en los que las tasas de desempleo y la precariedad del empleo creado se incrementa de forma exponencial. Un ejemplo de la importancia de dichas políticas se ha puesto claramente de manifiesto con motivo de la gravísima crisis económica que de forma general ha afectado a la economía mundial a partir del año 2007 y de la que con no pocas dificultades todavía se está saliendo. En este sentido y, por lo que se refiere a España, tanto el gobierno socialista, como posteriormente el gobierno del partido popular, adoptaron en su momento diversas iniciativas legislativas, que, con mayor o menor fortuna, iban dirigidas a frenar el proceso de destrucción de empleo provocada por la crisis económica y a activar el mercado de trabajo ${ }^{10}$.

\footnotetext{
${ }^{9}$ De este modo se quiere dar cumplimiento al mandato constitucional previsto en el artículo $40.1 \mathrm{CE}$, en virtud del cual "Los poderes públicos promoverán las condiciones favorables para el progreso social y económico (...). De manera especial realizarán una política orientada al pleno empleo”.

${ }^{10}$ Entre otras, cabe destacar la Ley 35/2010 de 17 de septiembre, de medidas urgentes para la reforma del mercado de trabajo (BOE de 18 de septiembre de 2010), Ley 3/2012, de 6 de julio, de medidas urgentes para la reforma del mercado laboral (BOE de 7 de julio) y la Ley 18/2014, de 15 de octubre, de aprobación de medidas urgentes para el crecimiento, la competitividad y la eficiencia (BOE de 17 de octubre de 2014).
} 
En este marco de actuación, entre los diferentes mecanismos que pueden resultar útiles a la hora de conseguir un incremento de las tasas de empleo se encuentran, sin duda, los correspondientes a la intermediación en el mercado de trabajo, los cuales se presentan como instrumentos facilitadores de la puesta en contacto de la oferta y la demanda de trabajo. Ahora bien, en las actuales economías tendencialmente globalizadas, donde cada vez se exige una mayor especialización y se reclama una mayor inmediatez o celeridad en la cobertura del puesto de trabajo, un número cada vez mayor de encuentros de ofertas y demandas de empleo traspasa no sólo el ámbito local o geográficamente más próximo, sino incluso las fronteras estatales; la fuerza de trabajo que no encuentra empleo satisfactorio en un determinado ámbito geográfico puede ser trasvasada a otro mercado de trabajo en el que existen requerimientos de mano de obra capaces de absorberla, por lo que los sistemas tradicionales de intermediación no siempre resultan efectivos en estos casos, produciéndose un abandono de tales mecanismos en favor de otros nuevos que "a priori" responden mejor a los requerimientos de los nuevos tiempos ${ }^{11}$.

En este contexto las NTICs han encontrado un amplio campo de acción y, se puede afirmar que la digitalización ha contribuido a dar un giro radical al ámbito de la intermediación, pasándose de una intermediación presencial o material a otra que podríamos denominar como intermediación digital, en el que cada vez es más frecuente la utilización de las redes sociales e internet para encontrar trabajo. A este respecto cabe señalar, entre otros, por ejemplo, el portal de empleo Infojobs.net, una de las empresas españolas de búsqueda de empleo más importantes y que ofrece ofertas de empleo en función de la zona geográfica y del sector, además de gestionar los curriculums enviados a las empresas; LinkedIn, la red social más utilizada en España según el III Informe-Adecco sobre Redes Sociales y Mercado de Trabajo ${ }^{12}$, la cual permite crear contactos profesionales, y donde además, se publican múltiples ofertas de empleo, es posible seguir a las empresas, así como participar en grupos de diferentes temáticas profesionales; Twitter, en donde existen cuentas, tipo @EmpleoyTrabajo, @Buscar_Trabajo, @Primerempleo, @Tecnoempleo, o @ Eujobsite, que tuitean ofertas de empleo, así como recomendaciones e información para facilitar la búsqueda a los usuarios; o Jobeeper, una aplicación que permite crear alertas de empleo, ver la descripción y los principales detalles de la oferta sin abandonar la app, enviar la oferta por email, y permite además inscribirse a la misma desde el móvil.

\footnotetext{
${ }^{11}$ Según datos ofrecidos por el Instituto Nacional de Estadística en el año 2017 tan sólo el 1,8\% de las personas trabajadoras en situación de desempleo encontraron trabajo por medio de los Servicios Públicos de Empleo. Vid. http://www.mitramiss.gob.es/es/estadisticas/anuarios/2017/index.htm [Fecha de consulta: 16/10/2018].

12 Vid. https://es.slideshare.net/InfoempleoSlide/iii-informe-infoempleo-adecco-sobre-redes-sociales-ymercado-de-trabajo [Fecha de consulta: 16/10/2018].
} 
Esta proliferación de nuevos sistemas de búsqueda de empleo o si se prefiere de facilitadores del contacto de oferta y demanda de trabajo, si bien puede resultar un vía interesante para cualquier persona demandante de empleo, lo es especialmente para aquellos colectivos que tradicionalmente tienen especiales dificultades para poder acceder a un trabajo, como es el caso del colectivo de las mujeres, que tanto en época de crisis, como de bonanza económica ven, año tras año, como sus niveles de empleo siguen siendo inferiores a los de los hombres. Un dato indicativo de esta situación lo encontramos en las cifras de desempleo del segundo trimestre de 2018, las cuales sitúan las tasas de desempleo global en un 15,28\%, si bien esos datos desagregados por sexo siguen siendo más desfavorables para las mujeres que para los hombres, ya que mientras la tasa de paro de los hombres se sitúa en el 13,72\%, el de las mujeres alcanza casi 4 puntos más situándose en el $17,08 \%{ }^{13}$.

Ahora bien, esta digitalización de la intermediación en el mercado de trabajo lleva a plantear diversas cuestiones relativas a su utilización y funcionamiento ya que entendemos que su implantación y desarrollo debe realizarse con garantías y, por tanto, sujetas a las mismas exigencias normativas que los sistemas tradicionales de intermediación.

Pues bien, el estudio de los diferentes mecanismos digitales y portales de internet que desarrollan su actividad en el marco de la intermediación en el mercado de trabajo permite constatar como bajo una aparente transparencia se encubre en realidad una estructura opaca a la hora de detectar el sujeto responsable de las obligaciones previstas en la normativa aplicable, siendo habitual la existencia de plataformas interpuestas que convierte en una aplicación informática a la propia entidad intermediadora, lo cual hace muy difícil en estos casos determinar las responsabilidades empresariales ${ }^{14}$.

De igual forma, las ofertas que podemos encontrar en los portales digitales también son muy poco transparentes, de manera que no es excepcional encontrar lo que podemos denominar "ofertas ciegas de trabajo", es decir ofertas de empleo que se anuncian sin incluir la más básica información sobre la condiciones de trabajo en las que se pretende desarrollar, como puede ser el tipo de contrato, el salario, la jornada o la formación requerida para el desarrollo del trabajo que se solicita. A tal efecto y, tal y como indica la doctrina ${ }^{15}$, se podría alegar que el proceso de intermediación es una fase previa a la

\footnotetext{
${ }^{13}$ Vid. http://www.ine.es/jaxiT3/Tabla.htm?t=4247 [Fecha de consulta: 16/10/2018].

${ }^{14}$ Chacartegui Jávega, C., "Incidencia de las nuevas TIC en la intermediación privada, el problema de la utilización de plataformas electrónicas para eludir derechos", XXIX Jornades Catalanes de Dret Social, Barcelona, 2018, ponencia multicopiada, p. 10.

${ }^{15}$ Ibid. p. 11.
} 
celebración del contrato de trabajo, momento en el que existe la obligación legal de comunicar a la persona trabajadora las principales condiciones de ejecución de la prestación de trabajo ${ }^{16}$. Sin embargo, también debe recordarse que el artículo 31 del RD Legislativo 3/2015, de 23 de octubre, por el que se aprueba el texto refundido de la Ley de Empleo (en adelante Ley de Empleo), establece que "la intermediación laboral tiene como finalidad proporcionar a los trabajadores un empleo adecuado a sus características y facilitar a los empleadores los trabajadores más apropiados s sus requerimientos y necesidades", por lo que si la oferta de trabajo anunciada en un portal digital carece o bien oculta información correspondiente a los elementos básicos del puesto de trabajo difícilmente puede valorarse adecuadamente si el perfil de una persona trabajadora se ajusta a los requerimientos de las empresas ${ }^{17}$.

Junto a la falta de transparencia que manifiestan en no pocas ocasiones los instrumentos digitales de intermediación en el mercado de trabajo, se suma, a nuestro parecer otro importante y preocupante problema como es el tratamiento realizado de los derechos fundamentales de la personas trabajadoras, una cuestión que afecta especialmente al colectivo de mujeres trabajadoras, ya que se constata que una de las vulneraciones que más frecuentemente puede apreciarse por parte de las agencias virtuales es la referida a la del principio de igualdad y no discriminación.

En este punto cabe recordar que el carácter virtual que pueda tener cualquier entidad que realice funciones de intermediación no exime del cumplimiento de las exigencias normativas requeridas en esta materia y, por tanto, la actuación de dichas entidades debe dar cumplimiento al mandato recogido en el artículo 35.1 de la Ley de Empleo según el cual "[1]os servicios públicos de empleo, sus entidades colaboradoras y las agencias de colocación en la gestión de la intermediación laboral deberán velar específicamente para evitar la discriminación tanto directa como indirecta en el acceso al empleo".

Pese a la referencia clara y taxativa al respeto a la igualdad y no discriminación en el acceso de empleo que realiza la Ley de Empleo, la cual también es posible encontrarla en otras normas laborales como el artículo 5 d) del RD 1796/2010, que regula las agencias de colocación o el artículo 17 ET, lo cierto es que los portales digitales se han convertido en terreno abonado para la vulneración del principio de igualdad y no discriminación por razón de sexo, de manera que no es excepcional la publicación de ofertas de empleo en las que se señala como colectivo destinatario uno de los dos sexos, reproduciéndose de nuevo los estereotipos de género en función del sector productivo. De este modo es bastante común la utilización del femenino cuando la oferta de empleo

\footnotetext{
${ }^{16}$ Vid. Artículo 8.5 ET y RD 1659/1998, de 24 de julio, en materia de información al trabajador sobre elementos esenciales del contrato de trabajo.

${ }^{17}$ Chacartegui Jávega, C., "Incidencia de las nuevas TiC...", cit. p. 11.
} 
es para desarrollar actividades de limpieza, cuidado de menores o personas mayores, representación, tratamientos estéticos o actividad textil ${ }^{18}$, mientras que si, por ejemplo, la prestación de servicios se enmarca dentro de actividades como la ingeniería, mecánica, pintura o el sector inmobiliario, el género utilizado en la oferta de trabajo tiende a ser el masculino ${ }^{19}$.

Estos ejemplos ponen de manifiesto que junto a las indudables ventajas que aporta la incorporación de las NTICs en los procesos de reclutamiento de personas trabajadoras a las plantillas de la empresas, también tales innovaciones se acompañan de importantes riesgos, por lo que si no queremos que se produzca un efecto boomerang resulta imprescindible no olvidar estos nuevos ámbitos de actuación a la hora de velar por el cumplimiento de la normativa laboral, siendo en este punto, a nuestro parecer indispensable, reforzar el papel de los servicios de la Inspección de Trabajo y Seguridad Social.

Junto a la actividad de intermediación, otro ámbito en el cual la NTICs pueden incidir de forma sustancial en el acceso al mercado de trabajo y, en especial en el acceso de la mujer es el referente a la formación. A este respecto resulta cada vez más frecuente la presencia en internet de recursos con capacidad de empoderar a las mujeres, lo que demuestra la concienciación social y la importancia de la presencia femenina en las redes. No se trata simplemente de un recurso de visibilidad, sino que constituye un medio que facilita el acceso a la mujer a la formación, al empleo y a los recursos que en definitiva incremente notoriamente las posibilidades de desarrollo de la persona ${ }^{20}$. Esta formación, además no sólo puede ir encaminada a facilitar el acceso a un primer empleo, sino que también puede ser un instrumento útil a la hora de fomentar la promoción profesional de las mujeres dentro de la empresa permitiendo una más fácil conciliación de la vida personal, familiar y profesional y, ayudando, de este modo a luchar con el denominado "techo de cristal" o "suelo pegajoso".

\footnotetext{
${ }^{18}$ En estos casos es habitual que la oferta de empleo se refiera a "empleada del hogar", "cuidadora", "niñera", "azafata", "peluquera" o "dependienta". Vid., por ejemplo, https://www.milanuncios.com/ofertas-de-empleo/ y https://www.infojobs.net/ofertas-trabajo [Fecha de consulta: 10/10/2018].

${ }^{19}$ Los términos "ingeniero", "técnico", "pintor", "agente inmobiliario", etcétera son los que de forma más común se utilizan a la hora de publicar las ofertas de empleo. Vid. por ejemplo, https://www.milanuncios.com/ofertas-de-empleo/ y https://www.infojobs.net/ofertas-trabajo [Fecha de consulta: 10/10/2018].

${ }^{20}$ DURAN BERNARDINO, M., "Nuevas tecnologías desde una perspectiva de género y de prevención desde el ámbito laboral”, Labour \& Law Issues, vol. 3, n 1, 2018, p. 6.
} 
Un último apunte en relación con la incidencia que pueden tener las NTICs en la incorporación de la mujer al mercado de trabajo viene referido al desarrollo del teletrabajo $^{21}$.

El teletrabajo ha sido definido por la Oficina Internacional del Trabajo como aquel trabajo "efectuado en un lugar distante de la oficina central o del centro de producción e implica una nueva tecnología que permite la separación y facilita la comunicación"22. En términos similares el Acuerdo Marco Europeo sobre Teletrabajo (en adelante AMET) se refiere al mismo como "una forma de organización y/o realización del trabajo utilizando tecnologías de la información en el marco de un contrato de trabajo o de una relación de trabajo, en la cual un trabajo que podría ser realizado en los locales de la empresa se efectúa fuera de ellos de forma regular" ${ }^{23}$. Es decir, se trata de un trabajo a distancia que se desarrolla principalmente por medio de instrumentos de carácter telemático, informático, etcétera.

Pues bien, justamente la posibilidad de prestar los servicios profesionales en un lugar distante o diferente a las instalaciones físicas de la empresa ofrece interesantes ventajas para la persona trabajadora, entre las que se encuentra la ampliación y mejora de las ofertas de trabajo, las cuales en algunos casos se hacen más accesibles, al no existir el impedimento de la localización/ubicación geográfica y, en otros casos, plantea la posibilidad de poder desarrollar algunos trabajos que de otro modo sería imposible por la flexibilidad horaria que ofrece el trabajo a distancia.

En este sentido se constata que con el teletrabajo la residencia de la persona trabajadora es un elemento irrelevante. Las distancias en este tipo de trabajo desaparecen y la no coincidencia o proximidad entre el domicilio de la persona trabajadora y el lugar de establecimiento de la empresa es indiferente, lo cual amplía el número de ofertas a las que puede acceder una persona demandante de empleo ${ }^{24}$. De igual forma la reducción/eliminación de desplazamientos o la posibilidad de organizar el trabajo de acuerdo con las preferencias o necesidades de la persona trabajadora, elementos caracterizadores del teletrabajo, facilita a muchas personas el acceso al mercado de trabajo, las cuales debido a dificultades inherentes a su persona o por razones personales

\footnotetext{
${ }^{21}$ Para un estudio sobre esta cuestión vid. Belzunegui ERASO, Á., Teletrabajo: estrategia de flexibilidad, CES, Madrid, 2002; Sellas Benvingut, R., El régimen jurídico del teletrabajo en España, Aranzadi, Cizur Menor (Navarra), 2001; SIERRA BENITEZ, E.M., El contenido de la relación laboral en el teletrabajo, CES Junta de Andalucía, Sevilla, 2011 y ThiBAult ARANDA, J., El teletrabajo. Análisis jurídico-laboral, CES, Madrid, 2001.

${ }^{22}$ Vid. Informe Oficina Internacional de Trabajo.

${ }^{23}$ Vid. Apartado 2 del Acuerdo Marco Europeo sobre el Teletrabajo, de 16 de julio de 2002.

${ }^{24}$ Estas ventajas ya se apuntaban por la Comisión Europea en el informe Telework (Telecommuting): Benefits. ACT Programme. DG XIII. Bruselas, 1999, p. 1 y 2.
} 
o familiares se ven excluidas del mercado de trabajo o tienen más dificultades para acceder, siendo un ejemplo paradigmático a este respecto el colectivo de mujeres ${ }^{25} \mathrm{y}$, en dentro de este colectivo en concreto, se nos antoja conveniente valorar el recurso del teletrabajo cuando la trabajadora víctima de violencia de género se vea imposibilitada para prestar servicios en su lugar habitual de trabajo para hacer efectiva su protección o su derecho a la asistencia social ${ }^{26}$.

De igual forma el teletrabajo puede favorecer la permanencia del empleo a las mujeres en situaciones de conflicto y especialmente en supuestos de acoso. En estos casos la empresa puede tomar la decisión de despedir a la persona acosadora o, alternativamente, proponerle una solución de alejamiento, como puede ser la de trabajar a distancia. En tal caso, se adoptan medias para, por un lado, resolver el conflicto de una forma menos traumática y no siempre segura para la empresa como puede ser un despido y, por otro lado, proteger a la víctima con el alejamiento de la persona acosadora. Ahora bien, no debe perderse de vista que la utilización del recurso del teletrabajo para supuestos tan graves como un acoso puede comportar efectos negativos si ante la situación de conflicto, el trabajo a distancia se ofrece a la víctima del mismo. La separación física y laboralmente a la víctima debe ser la última de las soluciones de carácter reparador que la empresa debe ofrecerle, salvo que sea ella misma la que lo solicite para alejarse del ambiente en el que ha sufrido el acoso ${ }^{27}$.

\section{El teletrabajo y la conciliación de la vida personal, familiar y profesional}

Junto a los mecanismos correctores que deben introducirse en el acceso al mercado de trabajo para la consecución de la igualdad efectiva entre hombres y mujeres en el

\footnotetext{
${ }^{25}$ Vid. MELla MÉNDEZ, L., "Configuración general del trabajo a distancia en el Derecho español” en MELla MÉNDEZ, L. (Directora). El teletrabajo en España: aspectos teórico-prácticos de interés, Wolters Kluwer España, S.A., Madrid, 2017, p. 43. En este sentido ya se manifestaban, entre otros organismos supraestatales, las instituciones europeas en diversos documentos que analizaban las oportunidades y los inconvenientes que acompañaban a la incorporación de las tecnologías de la información y comunicación en todos los ámbitos de la vida, valoraciones que siguen estando todas ellas vigentes. Vid., por ejemplo, Comisión Europea (1996). Vivir y trabajar en la sociedad e la información: prioridad para las personas. Libro Verde. Suplemento 3/96, Luxemburgo: Oficina de Publicaciones de las Comunidades Europeas, p. 27 y 28; European Comisión. Europe at the forefront of the global information society. COM (97), 3097, p. 4 a 6 y Comisión Europea (1998). La dimensión social y del mercado de trabajo de la sociedad de la información. Prioridad para las personas. Las próximas etapas, Luxemburgo: Oficina de Publicaciones de las Comunidades Europeas, p. 19 y 20.

${ }^{26}$ Vid. Villalba SÁNCHEZ, A., "Teletrabajo y responsabilidad social de la empresa” en Mella MÉNDEZ, L. y Villalba SÁNCheZ, A. (Editoras), Trabajo a distancia y teletrabajo. Estudios sobre su régimen jurídico en el derecho español y comparado, Thomson Reuters Aranzadi, Cizur Menor (Navarra), 2015, p. 77.

${ }^{27}$ Vid. MeLla MÉNDEZ, L., “Configuración general del trabajo a distancia...”, cit. p. 44.
} 
ámbito laboral, otro caballo de batalla al que habitualmente tienen que hacer frente las mujeres es el referido a la permanencia o estabilidad en su puesto de trabajo el cual, en no pocas ocasiones, puede verse en riesgo como consecuencia de la asunción por su parte de las responsabilidades familiares.

Tal y como se indica por la doctrina ${ }^{28}$, la incorporación de la mujer al trabajo "productivo" y "remunerado" supone la fractura o la necesaria superación de un sistema económico y jurídico que reproducía el modo histórico de organización social, patriarcal, en el que el sujeto protagonista de las relaciones de trabajo era el hombre, ocupado en las tareas productiva y remuneradas para el mantenimiento de su familia y donde la mujer era relegada al trabajo reproductivo no remunerado y a la gestión de las responsabilidades asociadas con los hijos y familiares y el hogar. Ahora bien, esa fractura o superación del modelo "masculino" de relaciones de trabajo no es todavía plena al no haberse erradicado la llamada doble presencia de la mujer, en el trabajo y en las tareas doméstico-familiares ${ }^{29}$, lo cual se traduce en una prolongación de la jornada diaria de las mujeres trabajadoras comportando la existencia de las denominadas "dobles y triples jornadas de trabajo" y el fracaso de la llamada "puerta giratoria" 30 , en tanto que las mujeres en la mayoría de los casos se sigue incorporando al mercado de trabajo sin abandonar su rol tradicional sino compatibilizándolo o superponiéndolo.

En este contexto la adopción de políticas públicas resulta nuevamente fundamental para conseguir eliminar la brecha de género en los cuidados existente en este momento, la cual pone en riesgo el trabajo de las mujeres y que sólo se cubrirá cuando al trabajar los hombres más horas en el hogar, las mujeres trabajen menos y así se alcance el equilibrio $^{31}$. A tal efecto, si mujeres y hombres asumieran con idéntica o similar intensidad las referidas "obligaciones familiares", esta circunstancia ya no podría ser tomada en consideración por el empresario a la hora de tratar de un modo distinto a ambos colectivos en relación con su integración, promoción y estabilidad laboral ${ }^{32}$.

\footnotetext{
${ }^{28}$ Vid. CASAS BaAmonde, M. E., “¿Derechos fundamentales específicos de las mujeres?”, Derecho de las Relaciones Laborales, $\mathrm{n}^{\circ}$ 1, 2017, p. 5.

${ }^{29}$ Vid. GUERRERo VizUETE, E., "Tiempo de trabajo y conciliación familiar: la excedencia por cuidado de hijos y familiares" en Romero Burillo, A. M. y Rodríguez Orgaz, C. (Coordinadores), Trabajo, Género e Igualdad, Thomson Reuters Aranzadi, Cizur Menor (Navarra), 2018, p. 242.

${ }^{30}$ Esta tesis fue acuñada en los años 60 por Young y Willmott, YounG, M. y WiLlmotT, M., Family and kinship in east London, Penguin Books, London, 1957, citado por GUERRERo VizUete, E., "Tiempo de trabajo y conciliación...", cit, p. 242.

${ }^{31}$ Díaz Martínez, C., "Introducción" en Díaz Martínez, C. y Simó-Noguera, (Coordinadores), Brecha salarial y brecha de cuidados, Tirant lo Blanch, Valencia, 2016, p. 13.

${ }^{32}$ Moreno GenÉ, J., "La suspensión del contrato de trabajo y la prestación por paternidad de los trabajadores por cuenta ajena diez años después de la aprobación de la ley de igualdad" en ROMERO Burillo, A. M. y RodríGUez Orgaz, C. (Coordinadores), Trabajo, Género..., cit. p. 143.
} 
Ahora bien, hasta hace relativamente poco tiempo, la articulación de los derechos laborales que promueven la conciliación de la vida personal, familiar y laboral ha girado en torno a los denominados "derechos de ausencia" "33, lo cual puede suponer para las mujeres consecuencias muy negativas, en tanto que son las principales usuarias de este tipo de medidas conciliadoras, ya que las misma comportan apartarse, aunque sea de forma parcial del mundo laboral, además de una merma económica que no sólo repercute en su retribución, sino que comportará efectos a largo plazo en el momento de percibir la correspondiente cobertura de protección social ${ }^{34}$, por ello se hace necesario que las medidas de conciliación no sólo promuevan y garanticen adecuadamente los períodos de ausencia que las tareas de cuidado de familiares puedan requerir, sino que se adopten medidas que permitan el establecimiento de una jornada laboral respetuosa con la vida familiar de las persona trabajadoras. "De nada sirve que (a las personas trabajadoras) se les ofrezca días de permisos y períodos de excedencia, que se agotan, si el día a día del trabajo imposibilita el compaginar con normalidad la vida laboral con la familiar" 35 .

En esta línea, la aprobación de la Ley Orgánica 3/2007, de 22 de marzo para la igualdad efectiva de mujeres y hombres ${ }^{36}$ (en adelante LOI) es sin duda un punto de inflexión desde la vertiente normativa en la lucha contra la discriminación que padecen las mujeres, erigiéndose como un instrumento especialmente idóneo para corregir las desigualdades en el ámbito laboral por motivos de género y para la promoción de una mayor corresponsabilidad entre hombres y mujeres en la asunción de las obligaciones familiares $^{37}$, lo cual se consigue sobre todo en la flexibilización de los tiempos de trabajo.

La LOI, como no podía ser de otra forma, incide en los tiempos de trabajo, introduciendo un conjunto de mejoras técnicas e incorporando significativas novedades respecto a permisos, vacaciones, suspensiones del contrato y medidas de protección social vinculadas a estos mismos derechos, que sin duda han supuesto un importante impulso a las políticas de conciliación, no obstante su despliegue a lo largo de todos

\footnotetext{
${ }^{33}$ BALLESTER PASTOR, M.A., "Conciliación laboral y familiar en la Unión Europea: rémoras, avances y nuevas líneas de tendencia en el ámbito de la corresponsabilidad", Revista de Derecho Social, no 51, 2010, p. 31 y ss.

${ }^{34}$ Charro BAENA, P., "La incidencia de la LOI en la ordenación del tiempo de trabajo: reducciones y permisos" en Romero Burillo, A.M. y Rodríguez Orgaz, C. (Coordinadores), Trabajo, Género ..., cit. p. 67.

${ }^{35}$ Vid. Informe Randstad sobre Calidad del trabajo en la Europa de los quince. Las políticas de conciliación, ESADE, Barcelona, 2004, pág. 100.

${ }^{36}$ BOE de 23 de marzo de 2007.

${ }^{37}$ Vid. Exposición de Motivos de la Ley Orgánicas 3/2007, de 22 de marzo para la igualdad efectiva de mujeres y hombres.
} 
estos años sigue poniendo de manifiesto la necesidad de nuevas acciones para que la corresponsabilidad de mujeres y hombres sea una realidad ${ }^{38}$.

Una de esas acciones adicionales es el teletrabajo, el cual desde hace ya mucho tiempo se viene apuntando como un instrumento idóneo para facilitar la conciliación de la vida personal, familiar y profesional, tanto a nivel doctrinal ${ }^{39}$, como a nivel normativo e institucional. En este sentido, cabe recordar que el AMET, en sus consideraciones generales ya hace expresa referencia a esta cuestión al señalar que "los interlocutores sociales consideran el teletrabajo a la vez como un medio de modernizar la organización del trabajo para las empresas y organizaciones de servicios públicos, y para los trabajadores reconciliar vida profesional y vida social (...)”.

Efectivamente, la incorporación de TICs que facilitan el trabajo fuera de las instalaciones de la empresa y, por tanto, la posibilidad de realizar el trabajo a distancia en las mismas condiciones que el resto de la plantilla que permanece físicamente en el centro de trabajo puede ser una alternativa viable que permita la permanencia de la persona trabajadora en la empresa cuando por circunstancias personales o familiares resulte incompatible con la prestación del trabajo en el lugar físico inicialmente fijado. En este sentido, el teletrabajo permite a las empresas retener a las personas trabajadoras que se plantean la posibilidad de dejar de trabajar, en especial las mujeres, que después de la suspensión por maternidad no pueden compatibilizar las tareas familiares y profesionales $^{40}$.

No obstante, las ventajas que se predican del teletrabajo a efectos de facilitar la conciliación de la vida personal, familiar y profesional los niveles de teletrabajo existentes en España son todavía bajos en relación con la Unión Europea y más aún respecto a los Estados Unidos ${ }^{41}$ y ello teniendo en cuenta que en los últimos años la generalización del uso de las NTICs por la población ha llevado mejorar su posición, pese a lo cual España sigue estando por debajo de la media europea ${ }^{42}$.

\footnotetext{
${ }^{38}$ Un amplio estudio sobre las medidas adoptadas por la LOI tanto a nivel laboral como de seguridad social, así como su despliegue en los últimos diez años se encuentra en ROMERo BURILlo, A. y Rodríguez Orgaz, C., Trabajo, Género e Igualdad, Thomson Reuters Aranzadi, Cizur Menor (Navarra), 2018.

${ }^{39}$ Vid. por todos Di MARTinO, V. y WIRTH, L., "Teletrabajo: un nuevo modo de trabajo y vida", Revista Internacional del Trabajo, n 109-4, 1990, p. 472.

${ }^{40}$ Ibid.

${ }^{41}$ Vid. FUNDACIÓN MASFAMILIA, El Libro Blanco del teletrabajo en España. Del trabajo a domicilio a los e-workers. Una recorrido por la flexibilidad espacial, la movilidad y el trabajo en remoto, Fundación Masfamilia, Madrid, 2015, p. 23 y ss.

${ }^{42}$ Vid. CES, Nuevos hábitos de consumo, cambios sociales y tecnológicos, Informe $n^{\circ} 4 / 2016$, Madrid: CES, 2012, p. 25.
} 
Los motivos que pueden justificar la poca utilización por parte de las empresas y las Administraciones Públicas del teletrabajo son diversos, pero sin duda entre ellos cabe destacar las reticencias que manifiestan tanto las organizaciones empresariales, como los sindicatos en que los empleados y las empleadas trabajen fuera de la empresa, con independencia de que la empresa pueda disponer de suficientes recursos estructurales para dotar de las infraestructuras adecuadas a fin de implantarlo ${ }^{43}$.

Tales reticencias a nuestro parecer derivan principalmente de la falta de una regulación concreta sobre teletrabajo, lo cual produce inseguridad jurídica y desprotección por mucha flexibilidad que se aporte a la organización del trabajo mediante este modo de trabajar $^{44}$. En este sentido cabe recordar que en nuestro ordenamiento jurídico laboral, en estos momentos sólo contamos con el artículo 13 ET que regula el trabajo a distancia $\mathrm{y}$, aunque dicha regulación fue actualizada y ampliada con motivo de la aprobación de la Ley 3/2012, resulta ciertamente parca, además de ser una regulación muy general que abarca cualquier tipo de trabajo a distancia, no sólo el teletrabajo, sin que sea posible en muchos casos poder acudir como alternativa a la regulación contenida en el ET, la cual resulta todavía más genérica. Por ello se hace necesaria una concreta regulación, entre otros aspectos, sobre ordenación del tiempo de trabajo, los descansos, la limitación de las jornadas de trabajo y su carácter voluntario ${ }^{45}$, la adecuación y medidas específicas en materia de salud laboral ${ }^{46}$ o sobre los sistemas de vigilancia y control $^{47}$.

Tampoco a nivel europeo contamos con una regulación lo suficientemente concreta sobre esta materia, ya que debemos recordar que la única regulación existente sobre teletrabajo es el AMET, por lo tanto, una norma que no obliga a los Estados miembro de la Unión Europea, sino que sólo es de obligado cumplimiento para los agentes sociales de los diferentes Estados de la Unión Europea en el momento en que se decida introducir por vía de la negociación colectiva la utilización del teletrabajo. Además, la necesidad de hacer viable una regulación a nivel europeo ha hecho necesario que el AMET adopte compromisos excesivamente genéricos, de manera que los derechos y obligaciones de las partes contratantes resultan muy poco precisos, quedando en manos del acuerdo concreto, pudiendo ser de esta forma la regulación muy diversa, tanto

\footnotetext{
${ }^{43}$ Vid. FUNDACIÓN MASFAMILIA, El Libro Blanco del teletrabajo en España ..., cit. p. 23 a 25.

${ }^{44}$ Vid. Quintanilla Navarro, R. Y., "Teletrabajo y conciliación de la vida personal, familiar y laboral", Revista del Ministerio de Empleo y Seguridad Social, nº 133, 2017, p. 362.

${ }^{45}$ Vid. SIERRA BENíteZ, E. M., "Trabajo a distancia y relación individual: aspectos críticos (I)" en Mella MÉnDEZ, L. (Directora), El teletrabajo en España ..., cit. p. 89 y ss.

${ }^{46}$ Mella Méndez, L. "La seguridad y salud en el teletrabajo" en Mella MÉndez, L. (Editora) y VILLALBA SÁNCHEZ, A.: Trabajo a distancia y teletrabajo..., cit. p. 171 y ss.

${ }^{47}$ Vid. CARDONA RUBERT, M.B., "Trabajo a distancia y relación individual: aspectos críticos (II)" en MELLA MÉNDEZ, Lourdes (Directora), El teletrabajo en España ..., cit. p. 141 y ss.
} 
respecto al Estado donde se implante, como a la empresa de la que se trate, sin que existan unos mínimos generales garantizados.

A partir de la existencia de una adecuada regulación del teletrabajo, con toda seguridad la implantación de este tipo de trabajo por parte de las empresas será mucho mayor. Ahora bien, convenimos con la doctrina ${ }^{48}$ que el hecho de que el teletrabajo permita compaginar de una forma equilibrada el cumplimiento de las obligaciones laborales y el desarrollo de la vida personal no debe llevar a considerar que la prestación de servicios a través de este modo constituya la mejor opción siempre. Su conveniencia dependerá de las funciones a desarrollar, del grado de autonomía que permitan las mismas y de que no se requiera la inexcusable presencia física de la persona trabajadora. A todo ello cabe añadir, además la posibilidad de plantear en el momento de su implantación el realizarlo sólo en una parte de la jornada laboral.

En todo caso el recurso al teletrabajo es un instrumento que cuenta con muchas potencialidades si se es capaz de dotarlo de una adecuada regulación, no solo para la persona trabajadora, sino también para las organizaciones empresariales si quieren conseguir el objetivo de ser consideradas socialmente responsables, en tanto que la empresa que ambicione alcanzar esta finalidad social con su actividad económica debe avanzar hacia un ideal de autorrealización personal de las personas trabajadoras que prestan los servicios para ella, combinando la eficiencia en el trabajo, la conciliación de la vida personal y laboral y la corresponsabilidad familiar ${ }^{49}$.

\section{El papel de las NTICs en los supuestos de acoso sexual y por razón de sexo: el ciberacoso}

Tal y como poníamos de relieve al inicio de este trabajo la rápida incorporación de las NTICs en nuestro día a día ha llevado a que su utilización se haya extendido no sólo al ámbito privado de las personas, sino también al ámbito de lo público. En este sentido, por ejemplo, no se entendería que un partido político ante un acontecimiento relevante no incorporara algún comentario en las redes sociales o no se tuvieran en cuenta los medios digitales en unas elecciones electorales.

Este impacto de las NTICs alcanza como no podía ser de otra manera también al marco de las relaciones de trabajo y ello ha comportado, entre otros efectos, que cada vez sea más habitual este tipo de comunicación entre empresa y personas trabajadoras a la hora de la ordenación de la actividad productiva, así como también entre las propias personas trabajadoras.

\footnotetext{
${ }^{48}$ Vid. Villalba SÁNCHEZ, A., “Teletrabajo y responsabilidad social...”, cit. p. 68 y 69.

${ }^{49}$ Ibid. p. 66.
} 
Es justamente esta proliferación de las comunicaciones a través de las NTICs y más concretamente su nefasta utilización por algunas personas, en especial de las redes sociales lo que ha llevado a la aparición de un nuevo fenómeno conocido con el término de "ciberacoso".

El ciberacoso, en términos generales, ha sido definido como aquel fenómeno en el que un individuo emplea una serie de conductas, para atacar, humillar, difamar, chantajear a otro, utilizando las tecnologías de la información y comunicación, principalmente internet y las redes sociales, pero con una progresiva incidencia a la través de los teléfonos móviles ${ }^{50}$.

Como se puede constatar, la definición de ciberacoso no dista mucho de la tradicional definición de acoso ${ }^{51}$, con la única excepción de la utilización de las NTICs para llevar a cabo dicha conducta, lo que lleva a que el acoso se introduzca en una nueva dimensión, hasta ahora desconocida y en la que parece que no existen límites.

Por tanto nos encontramos ante una problemática ya conocida pero que se produce en un nuevo escenario que amplía el ámbito de desarrollo de las conductas violentas en los lugares de trabajo y que en opinión de la doctrina resulta un campo especialmente abonado para el ejercicio de la violencia laboral de género, ya que la utilización de las NTICs, tienen como objetivo la dominación, la discriminación y, en definitiva el abuso de la posición de poder, posición que habitualmente ocupa el hombre ${ }^{52}$.

Este planteamiento teórico, desafortunadamente se ha visto concretado en la práctica de las empresas y, sobre esta cuestión es posible afirmar que el número de supuestos en los que las NTICs juegan un papel relevante a la hora sancionar disciplinariamente a una persona trabajadora por motivos de acoso se ha visto incrementado de forma importante en los últimos años, destacando especialmente las denominadas redes sociales, tipo Twitter y Facebook y el sistema de mensajería instantánea "WhastApp".

En este sentido y a título ilustrativo cabe señalar la STSJ de Cataluña de 17 de julio de $2012^{53}$ en la que se confirmará el acoso sexual que sufría una trabajadora por parte de un superior jerárquico de su empresa a partir de los comentarios incluidos en Facebook

\footnotetext{
${ }^{50}$ Vid. Vicente PaChes, F., "El ciberacoso: un fenómeno de violencia emergente en el ámbito de las relaciones de trabajo", Revista Aranzadi Instituciones, BIB 2017\634, p. 2.

${ }^{51}$ Para un estudio general sobre la actual configuración del acoso sexual y el acoso por razón de sexo en el ámbito laboral Vid. GiNés i FABRELLAS, A., "Acoso sexual y acoso por razón de sexo en la Ley Orgánica de Igualdad. Prevención y responsabilidad empresarial" en ROMERO BURILLO, A.M. y RodríGUEZ ORGAZ, C. (Coordinadores), Trabajo, Género..., cit. p. 295 y ss.

${ }^{52}$ Vid. Vicente PACHES, F., "El ciberacoso: un fenómeno de violencia...", cit. p. 4.

${ }^{53}$ JURI2012\297124.
} 
y el teléfono móvil. A este respecto la sentencia constata como durante más de un año la trabajadora fue víctima de los comentarios e insinuaciones del demandado tanto a través de mensajes de Facebook y de móvil que según consta en el informe emitido por los servicios de la Inspección de Trabajo eran constitutivos de un acoso sexual en la modalidad de chantaje que incluía la vulneración del derecho a la dignidad, lo cual llevó a la propuesta por parte de la Inspección de la determinación de que la baja de la trabajadora fuera de IT por contingencias profesionales.

Otro ejemplo en el que las NTICs son utilizadas como medio para llevar a cabo el acoso sexual lo encontramos en la STSJ del País Vasco de 13 de mayo de $2014^{54}$ en la que se aporta como prueba el envío que hacía el demandado por medio de "WhatsApp" de proposiciones sexuales a una trabajadora. En este supuesto se trata de una trabajadora empleada del hogar que prestaba sus servicios en el hogar y en el despacho profesional (consulta médica) del demandado y que denuncia a su empleador por tocamientos y acoso verbal sin consentimiento. Junto a la conductas físicas y comentarios obscenos, la trabajadora también recibía mensajes de "WhatsApp", con proposiciones sexuales y preguntas tales como el tipo de lencería que utilizaba.

Un supuesto similar al anterior se recoge en la STSJ de Castilla-La Mancha de 12 de febrero de $2015^{55}$, donde también se aportan las conversaciones mantenidas por las partes litigantes vía "WhatsApp" para fundamentar la conducta de acoso.

Para finalizar también puede destacarse, por un lado, la STSJ de Madrid de 8 de abril de $2016^{56}$, aunque en este caso el medio de prueba presentado es más tradicional al consistir en una serie de grabaciones audiovisuales. El supuesto analizado en la sentencia tiene su origen en la demanda de extinción del contrato de trabajo solicitado por la trabajadora ante el acoso sexual que estaba sufriendo por parte de su jefe y socio de la empresa, el cual queda probado, entre otros motivos, por la grabación realizada en la que se escucha que el demandado utiliza un lenguaje con connotaciones sexuales respecto a la demandante, lo cual puesto en relación con los informes médicos aportados, permitió fijar la existencia de un proceso de comentarios sexuales constantes y continuados hacia la trabajadora.

Y, por otro lado, la STSJ también de Madrid de 7 de noviembre de $2017^{57}$ que confirma la sentencia de instancia que declara despido disciplinario procedente el realizado por la empresa a uno de sus trabajadores por motivos de acoso sexual a una trabajadora

\footnotetext{
${ }^{54}$ JURL2014|230183.

${ }^{55}$ JUR 2015168340.

${ }^{56}$ AS 120161855.

${ }^{57}$ JURL2017\17976.
} 
realizado a través de notas y mensajes telefónicos de claro contenido amoroso durante un período de diez meses, siendo una conducta reiterada en el tiempo, rechazada por la destinataria, mantenida conscientemente por el acosador, a pesar de ser advertido de la necesidad de su cese y productores de una inequívoca tensión psicológica en la trabajadora.

Todos estos ejemplos pretenden poner de relieve los riesgos que se acompañan al mal uso de las NTICs y que es susceptible de lesionar o vulnerar múltiples derechos y libertades fundamentales de las personas trabajadoras, entre los cuales destacan derechos tan importantes como la dignidad de la persona, la intimidad, el derecho a la propia imagen, el derecho al honor, a la salud, la igualdad o la integridad física y moral. Por ello se hace cada vez más urgente que las empresas y las personas trabajadoras tomen conciencia de la importancia del uso responsable de las NTICs para lo cual resulta imprescindible contar con una formación y una especialización adecuada en esta materia y la profundización en una cultura preventiva sobre los riesgos en la red, una cuestión sobre la que todavía falta un gran concienciación y sensibilización.

\section{Bibliografía}

Belzunegui Eraso, A., Teletrabajo: estrategia de flexibilidad, CES, Madrid, 2002.

CARDONA Rubert, M., "La utilización de las redes sociales en el ámbito de la empresa", Revista de Derecho Social, nº 52, 2010.

CARdona Rubert, M. B., "Trabajo a distancia y relación individual: aspectos críticos (II)" en Mella MÉNDEZ, L. (Directora), El teletrabajo en España: aspectos teóricoprácticos de interés, Wolters Kluwer España, S.A., Madrid, 2017.

CASAS BAAmOndE, M.E., “Derechos fundamentales específicos de las mujeres?”, Derecho de las Relaciones Laborales, nº 1, 2017.

CES, Nuevos hábitos de consumo, cambios sociales y tecnológicos, Informe nº 4/2016, Madrid: CES, 2012.

Chacartegui Jávega, C., "Incidencia de las nuevas TIC en la intermediación privada, el problema de la utilización de plataformas electrónicas para eludir derechos", XXIX Jornades Catalanes de Dret Social, Barcelona, 2018. 
Charro Baena, P., "La incidencia de la LOI en la ordenación del tiempo de trabajo: reducciones y permisos" en Romero Burillo, A.M. y Rodríguez Orgaz, C. (Coordinador), Trabajo, Género e Igualdad, Thomson Reuters Aranzadi, Cizur Menor (Navarra), 2018.

CRUZ VILlalón, J., "Las transformaciones de las relaciones laborales ante la digitalización de la economía", Temas Laborales, n 118, 2016.

DEL REY GuAnter, S., "Sobre el futuro del trabajo: modalidades de prestaciones de servicios y cambios tecnológicos", IUSLabor, n² 2, 2017.

Di Martino, V. y WiRTh, L., "Teletrabajo: un nuevo modo de trabajo y vida", Revista Internacional del Trabajo, no 109-4, 1990.

Díaz Martínez, C., "Introducción" en DíAZ Martínez, C. y Simó-Noguera, (Coordinadores), Brecha salarial y brecha de cuidados, Tirant lo Blanch, Valencia, 2016.

DURAN BERnARDinO, M., "Nuevas tecnologías desde una perspectiva de género y de prevención desde el ámbito laboral”, Labour \& Law Issues, vol. 3, n 1, 2018.

FALGUERA BARÓ, M., "Nuevas tecnologías y trabajo (I): perspectiva contractual", Trabajo y Derecho, $\mathrm{n}^{\circ}$ 19-20, 2017.

FUNDACIÓN MASFAMILIA, El Libro Blanco del teletrabajo en España. Del trabajo a domicilio a los e-workers. Un recorrido por la flexibilidad espacial, la movilidad y el trabajo en remoto, Fundación Masfamilia, Madrid, 2015.

GiNÉS I FABRELLAS, A., “Acoso sexual y acoso por razón de sexo en la Ley Orgánica de Igualdad. Prevención y responsabilidad empresarial” en ROMERO BuRILLO, A.M. y RodríGuez Orgaz, C. (Coordinadores), Trabajo, Género e Igualdad, Thomson Reuters Aranzadi, Cizur Menor (Navarra), 2018.

Guerrero Vizuete, E., “Tiempo de trabajo y conciliación familiar: la excedencia por cuidado de hijos y familiares" en Romero Burillo, A.M. y RodríGuez OrGaz, C. (Coordinadores), Trabajo, Género e Igualdad, Thomson Reuters Aranzadi, Cizur Menor (Navarra), 2018.

Informe Randstad sobre Calidad del trabajo en la Europa de los quince. Las políticas de conciliación, ESADE, Barcelona, 2004. 
LlORENS EsPADA, J., "El uso de facebook en los procesos de selección de personal y la protección de los derechos de los candidatos", Revista de Derecho Social, nº 68, 2014.

Mella MÉndeZ, L., "Configuración general del trabajo a distancia en el Derecho español” en Mella MÉNDEZ, L. (Directora). El teletrabajo en España: aspectos teórico-prácticos de interés, Wolters Kluwer España, S.A., Madrid, 2017.

MerCAder Uguina, J. R., "La robotización y el futuro del trabajo", Trabajo y Derecho, $\mathrm{n}^{\circ} 27,2017$.

MoReno GenÉ, J., "La suspensión del contrato de trabajo y la prestación por paternidad de los trabajadores por cuenta ajena diez años después de la aprobación de la ley de igualdad" en Romero Burillo, A. M. y Rodríguez Orgaz, C. (Coordinadores), Trabajo, Género e Igualdad, Thomson Reuters Aranzadi, Cizur Menor (Navarra), 2018.

Nores TORRES, L. E., "Algunos puntos críticos sobre la repercusión de las redes sociales en el ámbito de las relaciones laborales: aspectos individuales, colectivos y procesales", Revista de Información Laboral, no 7, 2016.

QUINTANILLA NAVARRO, R. Y., "Teletrabajo y conciliación de la vida personal, familiar y laboral”, Revista del Ministerio de Empleo y Seguridad Social, nº 133, 2017.

Selma Penalva, A., "La información reflejada en las redes sociales y su valor como prueba en el proceso laboral. Análisis de los últimos criterios jurisprudenciales", Revista General del Derecho del Trabajo y de la Seguridad Social, nº 39, 2014.

Sellas Benvingut, R., El régimen jurídico del teletrabajo en España, Aranzadi, Cizur Menor (Navarra), 2001.

SIERRA BENITEZ, E. M., El contenido de la relación laboral en el teletrabajo, CES Junta de Andalucía, Sevilla, 2011.

SIERRA BENíTEZ, E. M., "Trabajo a distancia y relación individual: aspectos críticos (I)" en Mella MÉNDEZ, L. (Directora). El teletrabajo en España: aspectos teóricoprácticos de interés, Wolters Kluwer España, S.A., Madrid, 2017.

TAlEns Visconti, E. E., "La libertad de expresión de los sindicatos en las redes sociales", Aranzadi Social, versión electrónica, BIB 201512271. 
Thibault Aranda, J., El teletrabajo. Análisis jurídico-laboral, CES, Madrid, 2001.

Vicente PACHES, F., "El ciberacoso: un fenómeno de violencia emergente ene. Ámbito de las relaciones de trabajo", Revista Aranzadi Instituciones, BIB $2017 \backslash 634$.

Villalba SÁnCHEZ, A., "Teletrabajo y responsabilidad social de la empresa" en Mella MÉndez, L. y Villalba SÁnchez, A. (Editores), Trabajo a distancia y teletrabajo. Estudios sobre su régimen jurídico en el derecho español y comparado, Thomson Reuters Aranzadi, Cizur Menor (Navarra), 2015. 